\title{
Evaluación de Mezclas Ternarias de Petrodiésel, Biodiésel de Palma y Oleorresina de Copaiba en una Planta Eléctrica
}

\author{
Anny A. Espitia*, Arnoldo E. Delgado y William A. Aperador \\ Facultad de Ingeniería, Universidad Militar Nueva Granada, carrera 11 No. 101-80 Bogotá - Colombia. \\ (e-mail: anny.espitia@unimilitar.edu.co; arnoldo.delgado@unimilitar.edu.co; william.aperador@unimilitar.edu.co) \\ ${ }^{*}$ Autor a quien debe ser dirigida la correspondencia
}

Recibido Nov. 27, 2018; Aceptado Ene. 17, 2019; Versión final Feb. 27, 2019, Publicado Ago. 2019

\begin{abstract}
Resumen
Se analiza el desempeño de mezclas de petrodiésel, biodiésel de palma y oleorresina de copaiba refinada, como combustible en un generador eléctrico. Los ensayos fueron realizados en una planta eléctrica provista con un sistema de alimentación secundario para monitorear el desempeño de las mezclas y el combustible de referencia en condiciones de carga plena. Se estudió la influencia del porcentaje de oleorresina en las mezclas sobre el consumo de combustible, la potencia entregada y la emisión de gases de escape. Se estableció una disminución de hasta el $19 \%$ en la potencia generada por la planta respecto a la energía suministrada por el motor operado con diésel. En relación a la emisión de gases, la adición de oleorresina de copaiba refinada al combustible, redujo la concentración de $\mathrm{CO}_{\text {y SO}}$ en los gases de escape. De acuerdo a los resultados, la incorporación de oleorresina de copaiba al diésel comercial, podría constituir una opción ambientalmente amigable. Sin embargo, el aumento en la viscosidad del combustible limitaría su uso en aplicaciones automotrices.
\end{abstract}

Palabras claves: oleorresinas; copaiba; biocombustibles; emisión de gases; motor diésel

\section{Evaluation of Mixtures of Petrodiesel, Palm Biodiesel and Copaiba Oleoresin in an Electrical Plant}

\begin{abstract}
The performance of mixtures of petrodiesel, palm biodiesel and refined copaiba oleoresin as fuel in an electric generator is analyzed. Tests were carried out in a power plant provided with a secondary feeding system to monitor the performance of the mixtures and of the reference fuel under full load conditions. Influence of percentage of oleoresin in mixtures on fuel consumption, power delivered and emission of exhaust gases were studied. A decrease of up to $19 \%$ was established in power generated by plant with respect to energy supplied by the diesel-operated engine. In relation to gases emission, the addition of refined copaiba oleoresin to fuel, reduces concentration of $\mathrm{CO}$ and $\mathrm{SO}_{2}$ in exhaust gases. According to the results, incorporation of copaiba oleoresin to commercial diesel could be an environmentally friendly option. However, increase in viscosity of fuel could limit its use in automotive applications.
\end{abstract}




\section{INTRODUCCIÓN}

La problemática energética a nivel mundial derivada de la creciente demanda de energía y el agotamiento de las reservas de petróleo, así como los problemas de contaminación ambiental asociados al uso de combustibles de origen fósil, tales como la emisión de nitratos y sulfatos, originados por la oxidación de compuestos NOx y SOx, y la emisión de hidrocarburos policíclicos aromáticos a la atmósfera, ha generado un interés creciente en la búsqueda de alternativas a los combustibles derivados del petróleo por combustibles respetuosos del medio ambiente (Leevijit et al., 2017; Morone y Cottoni, 2016; Reddy et al., 2016; Habibullah et al., 2015; Sitepu et al., 2014). Colombia no es ajena a esta situación, y al igual que la posición adoptada por otros países, sabiendo que los recursos naturales son limitados y que la tasa de consumo supera la de producción, pretende avanzar hacia un escenario que no sea sólo amigable con el medio ambiente sino también sostenible, por ello se ha propuesto trabajar en sustituir o al menos reducir el uso de combustibles fósiles.

En este contexto se ha buscado migrar al uso de biocombustibles, que se caracterizan por tener una combustión más limpia y se consideran renovables al provenir de materiales vegetales que se pueden reponer indefinidamente. Entre los tipos de biocombustibles alternativos a aquellos de origen fósil se encuentran los bioalcoholes, el biodiésel y los aceites vegetales. Estos últimos poseen bajos contenidos de azufre y compuestos aromáticos, altos puntos de inflamación y poder lubricante; sin embargo, su viscosidad elevada ha limitado su uso como combustible. Emplear aceite vegetal, aunque puede constituir una opción ambientalmente amigable para su uso en aplicaciones automotrices, no ha tenido gran acogida debido a los problemas derivados de su uso, tales como taponamiento de filtros e inyectores, combustión incompleta y depósitos de carbón en la cámara de combustión, entre otros (Qi et al., 2017; Suh and Lee, 2016; Corsini et al., 2015). Procesos como la transesterificación, pirolisis, micro-emulsión y mezclas con combustible diésel, son algunas técnicas empleadas para reducir la viscosidad de los aceites vegetales (Atmanli et al., 2015; Rakopoulos et al., 2014).

En la literatura se encuentran estudios sobre la obtención de biocombustibles a partir de oleorresinas como la extraída a partir del árbol de copaiba, la cual, aunque tradicionalmente ha sido empleada como analgésico, anti-inflamatorio y anti-leishmaniasis (Dhorm Pimentel de Moraes et al., 2018; Tobouti et al., 2017; Bardají et al., 2016), puede ser considerada como posible sustituto del combustible tradicional, como se menciona en los estudios de Veiga Junior y Pinto (2002), Chen et al. (2009) y da Silva et al. (2010), quienes señalan el potencial de su uso directamente como combustible o en mezclas con diésel, despertando el interés en la investigación de las características de la oleorresina de copaiba para la formulación de biocombustibles. En un trabajo previo, se estableció qué a través de un proceso de refinado químico con una base fuerte, la viscosidad en la oleorresina puede disminuir drásticamente, haciendo más factible su uso como combustible alternativo; este hallazgo fue el punto de partida para realizar la presente investigación (Espitia et al., 2018). En este estudio se evaluó el desempeño como combustible de mezclas de oleorresina de copaiba refinada y diésel. Variables como la potencia, consumo de combustible y la emisión de gases, fueron comparadas a fin de establecer los beneficios que podría ofrecer la incorporación de la oleorresina en la formulación de combustibles.

\section{MATERIALES Y MÉTODOS}

Se utilizó diésel comercial ( $91,9 \%$ petrodiésel - 8,1\% de biodiésel de palma) y oleorresina de copaiba (OC) extraída directamente de árboles de Copaifera reticulata en la amazonia brasilera (viscosidad cinemática 53,3 $\left.\mathrm{mm}^{2} / \mathrm{s}\right)$.

\section{Refinado}

Inicialmente la $\mathrm{OC}$ fue tratada con una disolución de $\mathrm{NaOH}$ al $10 \%$ durante 10 minutos a $30^{\circ} \mathrm{C}$. Transcurrida la reacción, la temperatura de la mezcla fue elevada hasta $75^{\circ} \mathrm{C}$ para separar las fases. Posteriormente la $\mathrm{OC}$ neutralizada fue lavada con agua hasta alcanzar un $\mathrm{pH}$ neutro. Finalmente, el exceso de humedad fue retirado con la adición de $\mathrm{Na}_{2} \mathrm{SO}_{4}$ anhidro en proporción 4:1 respecto al aceite.

\section{Elaboración de mezclas}

Se prepararon tres mezclas ternarias (C5, C10 y C20) de petrodiésel/biodiésel de palma y oleorresina de copaiba refinada $(\mathrm{OCR})$, como se muestra en la Figura 1. El combustible comercial fue mezclado directamente con 5,10 y $20 \%$ en volumen de OCR. En adelante la palabra diésel, hará referencia a la mezcla petrodiésel/biodiésel de palma (C0). 


\section{Caracterización de las mezclas.}

Se determinaron la densidad, viscosidad (ASTM D2983 -04), punto de fluidez (ASTM D97-05c), punto de inflamación (ASTM D92-05a) y corrosión en lámina de cobre (ASTM D130-12) aplicando los procedimientos descritos en las normas.

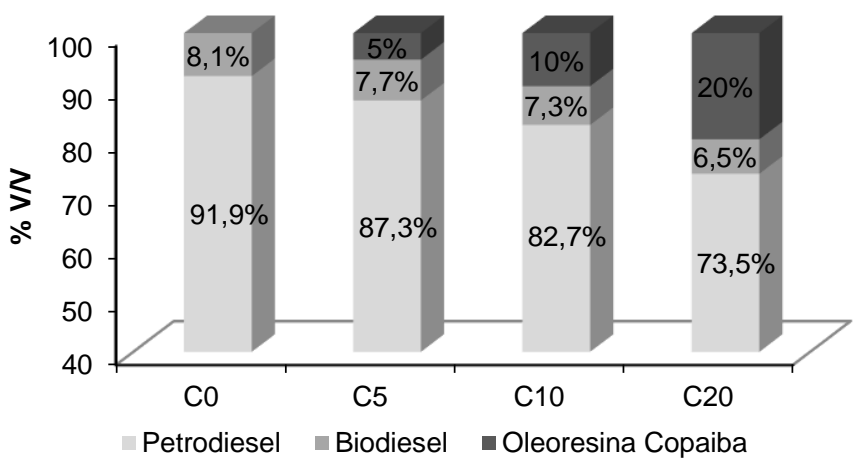

Fig. 1: Composición porcentual de las mezclas a evaluar.

\section{Obtención de espectros FTIR-HART}

Para el análisis de la composición estructural de las mezclas por espectroscopia infrarroja, se utilizó un espectrómetro Nicolet iS10 (Thermo Fisher Scientific) operado en modo transformada de Fourier, provisto de un accesorio de reflectancia total atenuada horizontal (HATR) y cristal de ZnSe. Los espectros fueron obtenidos con una resolución de $4 \mathrm{~cm}^{-1}$ en un rango espectral de $4000-650 \mathrm{~cm}^{-1}, 32$ barridos y procesados con el software omnic 9.1.27.

\section{Condiciones de operación y procedimiento de los ensayos}

Los ensayos fueron realizados en la ciudad de Bogotá D.C a una altura de $2650 \mathrm{msnm}$, en un generador eléctrico Diésel Hi-Force 7HP modelo DG3500CL/E de encendido eléctrico, refrigerado con aire forzado y operado a plena carga. Las especificaciones técnicas del generador se presentan en la Tabla 1.

Tabla 1: Especificaciones técnicas de la planta eléctrica.

\begin{tabular}{lc}
\hline \multicolumn{1}{c}{ Ítem } & Especificación \\
\hline Motor & \\
Tipo & 4 tiempos \\
Sistema de inyección & Directa \\
Número de cilindros & 1 \\
Relación de compresión & 20 \\
Potencia del motor $(\mathrm{hp})$ & 6 \\
Consumo especifico de combustible $(\mathrm{g} / \mathrm{kWh})$ & $\leq 292,5$ \\
Velocidad nominal $(\mathrm{rpm})$ & 3600 \\
Capacidad cubica del cilindro $\left(\mathrm{cm}^{3}\right)$ & 296 \\
Generador & \\
Potencia nominal $(\mathrm{kW})$ & 3,3 \\
Frecuencia nominal $(\mathrm{Hz})$ & 60 \\
Voltaje $(\mathrm{V})$ & $110-120$ \\
\hline
\end{tabular}

Inicialmente el generador fue energizado empleando el diésel proveniente del tanque principal, transcurridos 3 min de operación, las cargas fueron conectadas de manera gradual a la planta. El sistema se dejó estabilizar durante $20 \mathrm{~min}$, hasta que la temperatura se mantuvo estable en $96^{\circ} \mathrm{C}$. Durante toda la operación la temperatura de la superficie del motor fue monitoreada empleando un termómetro tipo termocupla ModernL ${ }^{\circledR}$.

Una vez estabilizado el sistema, el suministro de combustible se realizó a través del sistema de alimentación secundario acoplado a la planta eléctrica cuyo tanque es una probeta externa de $1000 \mathrm{~cm} 3$ (Fig. 2), con el fin de evaluar el desempeño del combustible de referencia (C0) y las mezclas C5, C10 y C20. Al finalizar cada ensayo, la válvula secundaria fue cerrada. La alimentación de combustible continúo desde el tanque principal y el sistema se dejó estabilizar durante 15min después de cada prueba. En todos los casos se analizaron las características de combustión y emisión de gases. 


\section{Determinación del consumo de combustible}

El consumo fue medido empleando un cronometro digital. En cada intervalo de tiempo se registró el volumen de combustible en el tanque de alimentación secundario.

$\mathrm{R}_{1}$

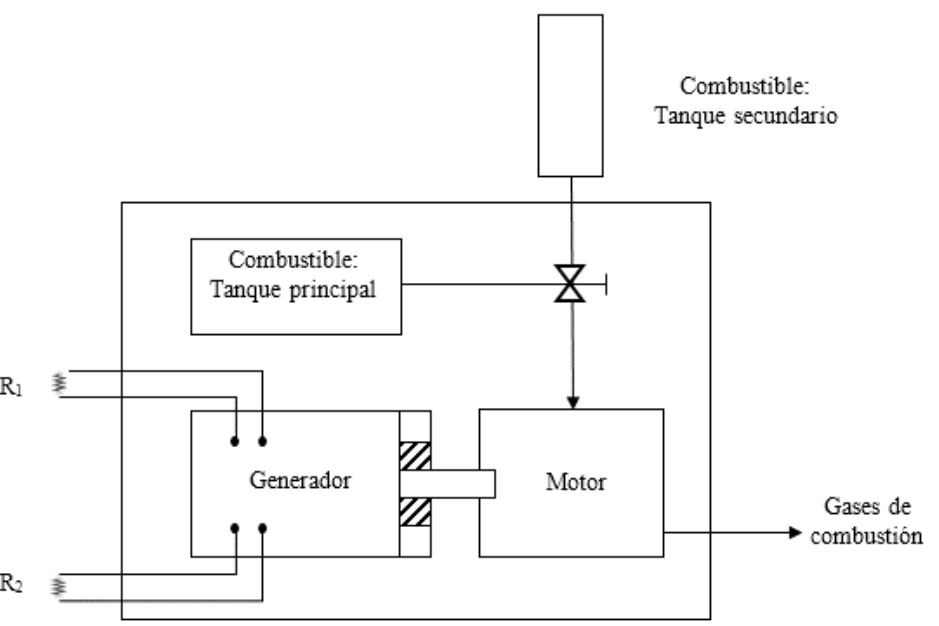

Fig. 2: Montaje empleado para los ensayos.

Medición de la potencia

La potencia entregada por el generador se calculó como la razón entre el amperaje promedio para cada mezcla y el voltaje nominal del equipo. La corriente entregada por el generador fue medida empleando una pinza amperimétrica.

Análisis de los gases de combustión

Se empleó un analizador multifuncional de gases serie GT-2000 para establecer la concentración de monóxido de carbono $(\mathrm{CO})$, dióxido de carbono $\left(\mathrm{CO}_{2}\right)$, dióxido de azufre $\left(\mathrm{SO}_{2}\right)$ e hidrocarburos no quemados $\left(\mathrm{C}_{\mathrm{x}} \mathrm{H}_{\mathrm{y}}\right)$ en los gases de combustión del diésel y sus mezclas con OCR. Se realizaron tres mediciones para cada gas en intervalos de 4 minutos.

\section{Análisis estadístico}

La influencia del porcentaje de oleorresina de Copaiba sobre la concentración de $\mathrm{C}_{\mathrm{x}} \mathrm{H}_{\mathrm{y}}, \mathrm{CO}, \mathrm{CO}_{2}, \mathrm{SO}_{2}$ en los gases de combustión de las mezclas OCR/diésel, fue estudiada a través de un análisis de varianza de una vía empleando el programa Statgraphics ${ }^{\circledR} 5.0$ con un nivel de confianza del $95 \%$. La comparación de medias se realizó a través de pruebas de rango múltiple con el método HSD Tukey.

\section{RESULTADOS Y DISCUSIÓN}

Después del proceso de refinado, la oleorresina de copaiba presentó una viscosidad cinemática de 11,2 $\mathrm{mm}^{2} / \mathrm{s}$, alcanzando una disminución del $79 \%$ respecto a la oleorresina cruda. La densidad y viscosidad de las mezclas aumentaron gradualmente con el incremento del porcentaje de oleorresina de copaiba refinada.

Tabla 2: propiedades fisicoquímicas del diésel y sus mezclas con oleorresina de copaiba refinada.

\begin{tabular}{lccccc}
\hline \multicolumn{1}{c}{ Propiedad } & $\mathrm{C} 0$ & $\mathrm{C} 5$ & $\mathrm{C} 10$ & $\mathrm{C} 20$ & NTC 1438 \\
\hline Densidad $40{ }^{\circ} \mathrm{C}(\mathrm{g} / \mathrm{ml})$ & 0,840 & 0,846 & 0,850 & 0,858 & - \\
Viscosidad cinemática $40{ }^{\circ} \mathrm{C}\left(\mathrm{mm}^{2} / \mathrm{s}\right)$ & 3,3 & 3,5 & 3,6 & 3,8 & $1,9-5$ \\
Punto de inflamación $\left({ }^{\circ} \mathrm{C}\right)$ & 59 & 65 & 58 & 68 & 52 min. \\
Punto de fluidez $\left({ }^{\circ} \mathrm{C}\right)$ & 0 & -3 & -3 & -3 & 3 máx. \\
Corrosión lámina de cobre & $1 \mathrm{~A}$ & $1 \mathrm{~A}$ & $1 \mathrm{~B}$ & $1 \mathrm{~B}$ & 2 máx. \\
\hline
\end{tabular}

Con la adición de OCR en concentraciones de 5, 10 y 20\% vol. al diésel, además de reducir hasta $73,5 \%$ el porcentaje de petrodiésel en el aceite, se mejoró la fluidez del combustible a baja temperatura. El índice de fluidez de las mezclas se mantuvo en $-3^{\circ} \mathrm{C}$ independientemente de la concentración de oleorresina añadida (Tabla 2). En contraste con lo reportado por Ali et al. (2016), quienes establecieron que la incorporación de biodiésel de palma al diésel convencional, afectó negativamente las propiedades de flujo en frio de las 
mezclas, obteniendo una variación en el punto de fluidez de $-14^{\circ} \mathrm{C}$ a $8^{\circ} \mathrm{C}$ aproximadamente, con la incorporación de $30 \%$ vol. de biodiésel al combustible de referencia; el uso de OCR para la formulación de biocombustibles podría contribuir en la mejora de la fluidez del combustible a bajas temperaturas, al disminuir como en este caso, la temperatura a la que podría fluir el combustible y por ende los problemas de taponamiento de filtros y líneas de combustible, usualmente asociados a un alto contenido de metil esteres de ácidos grasos en el biodiésel (Ali et al.,2014).

La adición de 5 y $20 \%$ de OCR al diésel, incrementó en 10 y $15 \%$ el punto de inflamación del aceite combustible. De acuerdo a la norma ASTM D130 el efecto corrosivo del azufre en el aceite, tanto para el diésel como para sus mezclas con OCR, sobre aleaciones de cobre fue clasificada como grado $1 \mathrm{~A}$ para C0 y C5 y grado 1B para C10 y C20 (Tabla 2). En todos los casos se mantuvo un leve de desgaste en la lámina de cobre, con un ligero cambio de coloración, al incrementar la concentración de OCR a 10 y 20\%. De acuerdo a los resultados, las propiedades fisicoquímicas de las mezclas ternarias se encuentran dentro de los valores permitidos según la Norma Técnica Colombiana NTC 1438.

\section{Análisis FTIR-HART}

El análisis del espectro infrarrojo de la mezcla C0 permitió corroborar que corresponde a una mezcla petrodiésel - biodiésel, como se confirma por la presencia de bandas de absorción propias de los esteres metílicos en el aceite.

Para todos los espectros son comunes las bandas de absorción entre 2850 y $2960 \mathrm{~cm}^{-1}$, la vibración del grupo carbonilo $(C=O)$ en $1746 \mathrm{~cm}^{-1}$ característica de los esteres, la deformación $C=C$ de los dobles enlaces olefínicos en $1644 \mathrm{~cm}^{-1}$, bandas centradas en 1376 y $1457 \mathrm{~cm}^{-1}$, bandas en la región 1300-1100 cm-1 originadas por la deformación axial asimétrica de los enlaces $C$-O y la flexión fuera del plano de los enlaces $=C-H$ de las olefinas en la región comprendida entre $700-1000 \mathrm{~cm}^{-1}$ (Fig. 3).

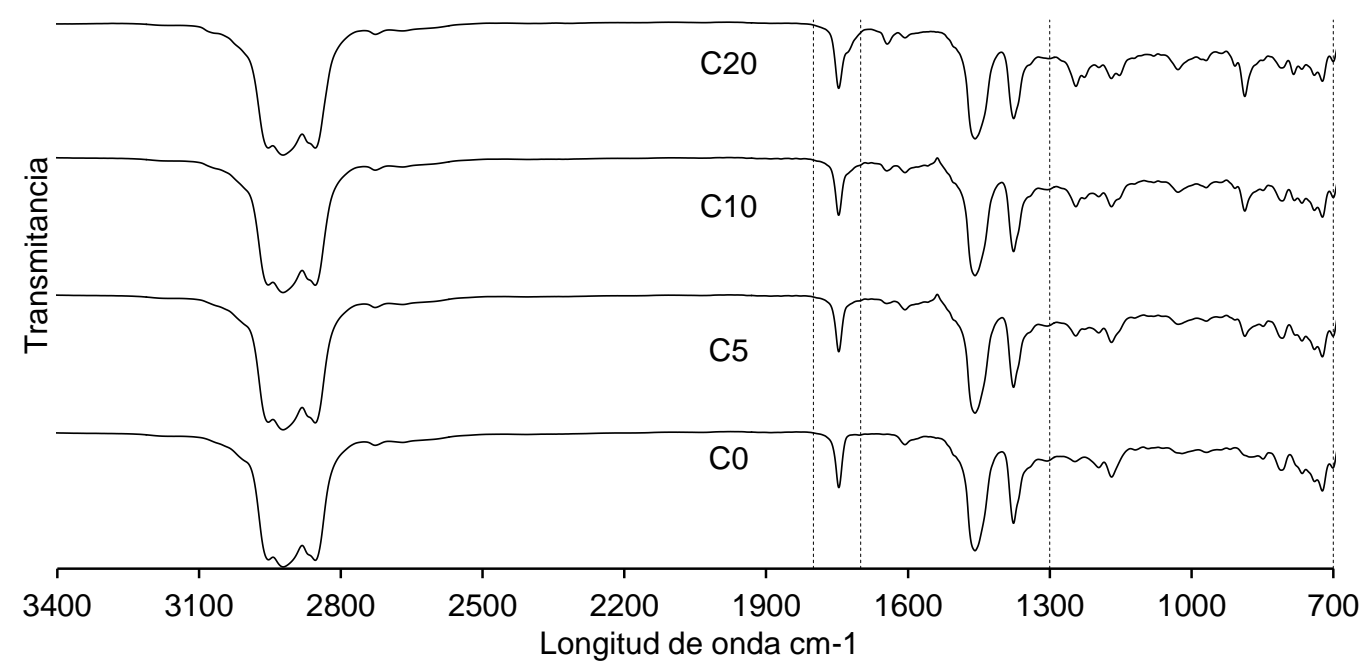

Fig. 3: Espectros FT-IR para el diésel y las mezclas ternarias.

Sin embargo, al contrastar los espectros de las mezclas OCR/diésel, se encontraron diferencias en los niveles de absorbancia de los grupos funcionales asociados a la vibración de los esteres metílicos y las olefinas. A medida que aumenta la concentración de OCR en las mezclas, disminuye la transmitancia de los enlaces asociados a estos grupos funcionales, aspecto que está directamente relacionado con el aumento de la concentración de los mismos en la mezcla (Lafont et al., 2011).

\section{Consumo de combustible}

El consumo de combustible se mantuvo estable, independientemente del tipo de mezcla empleada para operar el generador (Fig. 4). Respecto a la temperatura de la superficie del motor, con el uso de las mezclas ternarias se mantuvo una temperatura constante de $100^{\circ} \mathrm{C}$, dos grados por encima de la temperatura alcanzada con el diésel, variación atribuida al calentamiento del equipo durante el tiempo de operación. En general, se infiere que el tipo de mezcla no tuvo influencia sobre el consumo de combustible y la temperatura de la superficie del motor. 


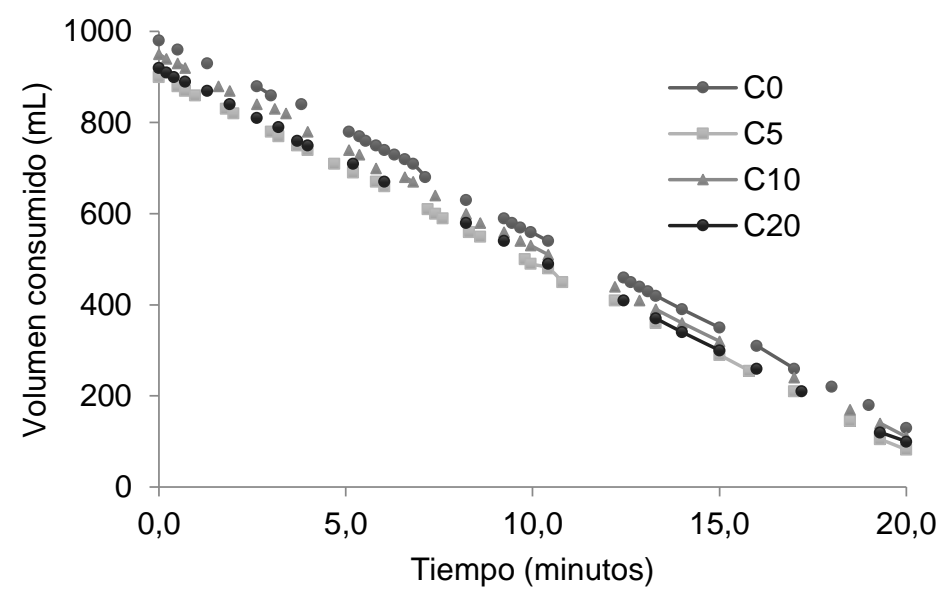

Fig. 4: Consumo de combustible.

\section{Potencia}

La adición de OCR al diésel ocasionó pérdidas de potencia en el motor. Al operar el motor con las mezclas C5, C10 y C20, se presentaron perdidas de potencia del 9, 19 y 16\% respectivamente, en relación a la potencia entregada por el generador operado con diésel (Fig. 5). Esto puede ser atribuido al aumento en la viscosidad de las mezclas (Tabla 2), aspecto que afecta la inyección y pulverización del combustible, e incrementa la energía necesaria para bombear el aceite, reduciendo la potencia entregada por la planta eléctrica (Altun y Lapuerta, 2014). Estos resultados concuerdan con lo reportado por Chuah et al. (2015), quienes determinaron que el aumento en la viscosidad de mezclas de biodiésel de aceite de cocina usado y diésel, ocasionó una disminución progresiva de hasta 6,7\% en la potencia del motor a medida que aumentaba el contenido de biodiésel en la mezcla hasta el $50 \%$.

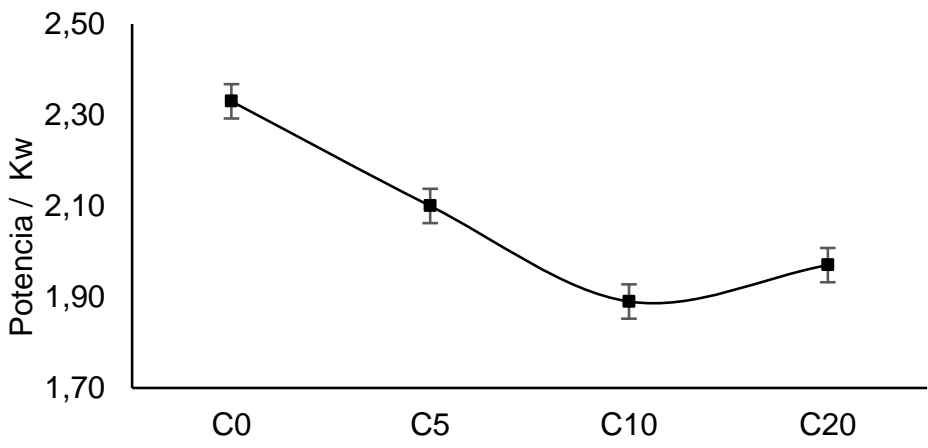

Fig. 5: Potencia entregada por el generador.

\section{Gases de combustión}

No fue posible establecer una tendencia sobre el comportamiento de las emisiones de $\mathrm{C}_{\mathrm{x}} \mathrm{H}_{\mathrm{y}}$, debido a las fluctuaciones en los niveles de emisión en función del tipo de combustible (Fig. 6). Estadísticamente el tipo de mezcla ternaria no influyó de forma significativa sobre la concentración de $\mathrm{C}_{x} \mathrm{H}_{\mathrm{y}}$ en los gases de combustión (Tabla 3).

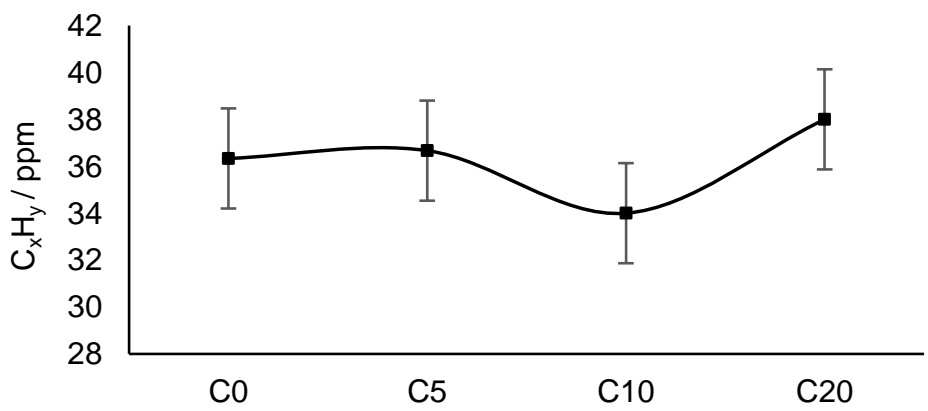

Fig. 6: Concentración de hidrocarburos no quemados en los gases de escape. 
Tabla 3: Análisis de varianza para la emisión de $\mathrm{C}_{x} \mathrm{H}_{\mathrm{y}}$ en función del tipo de combustible.

\begin{tabular}{cccccc}
\hline Fuente & Suma de Cuadrados & Gl & Cuadrado Medio & Razón-F & Valor-P \\
\hline Entre grupos & 24,9167 & 3 & 8,30556 & 3,11 & 0,0883 \\
Intra grupos & 21,3333 & 8 & 2,66667 & & \\
Total (Corr.) & 46,25 & 11 & & & \\
\hline
\end{tabular}

La adición de OCR al aceite combustible influyo significativamente sobre los niveles de emisión de compuestos de azufre (Tabla 4). Al reducir el contenido de diésel en las mezclas ternarias, disminuyó la concentración de azufre en aceite y por ende la emisión de $\mathrm{SO}_{2}$ en los gases de combustión. A partir del análisis estadístico, se estableció una disminución del $11 \%$ en la concentración de $\mathrm{SO}_{2}$ en los gases de escape producto de la combustión de las mezclas diésel/ OCR al 20\%, respecto a las emisiones con el combustible de referencia (Fig. 7).

Tabla 4: Análisis de varianza para la emisión de $\mathrm{SO}_{2}$ en función del tipo de combustible.

\begin{tabular}{llclcc}
\hline Fuente & Suma de Cuadrados & Gl & Cuadrado Medio & Razón-F & Valor-P \\
\hline Entre grupos & 219,583 & 3 & 73,1944 & 6,55 & 0,0151 \\
Intra grupos & 89,3333 & 8 & 11,1667 & & \\
Total (Corr.) & 308,917 & 11 & & & \\
\hline
\end{tabular}

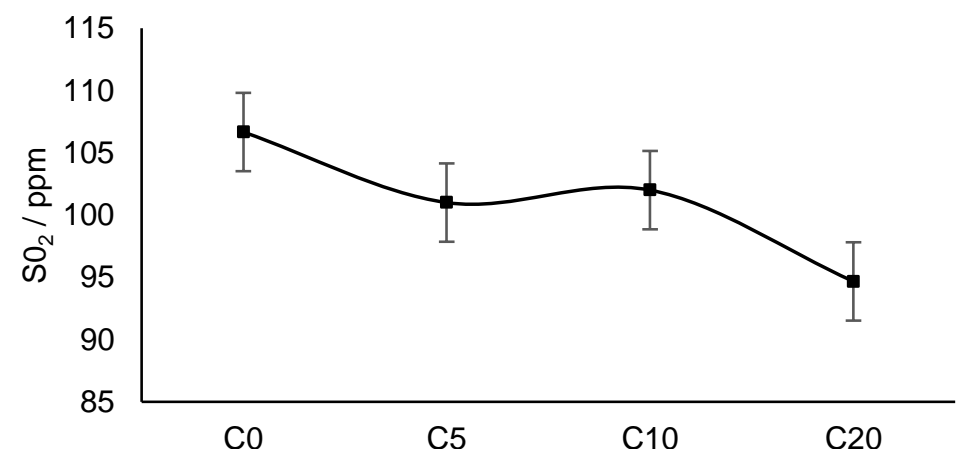

Fig. 7: Concentración de dioxido de azufre en los gases de escape.

De acuerdo con la prueba de rangos múltiples, la concentración promedio $(\overline{\mathrm{x}})$ de $\mathrm{SO}_{2}$ en los gases de combustión de las mezclas C5 y C10 presentó un comportamiento homogéneo y el contraste de medias entre C0 y C20 es estadísticamente significativo (Tabla 5).

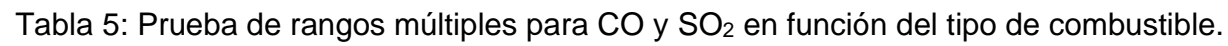

\begin{tabular}{|c|c|c|c|c|c|}
\hline \multirow{2}{*}{\multicolumn{2}{|c|}{ Combustible }} & \multicolumn{2}{|c|}{$\mathrm{CO}$} & \multicolumn{2}{|c|}{$\mathrm{SO}_{2}$} \\
\hline & & $\overline{\mathrm{x}}(\% \mathrm{v})$ & gh & $\overline{\mathrm{x}}(\mathrm{ppm})$ & \multirow{2}{*}{$\frac{g h}{x}$} \\
\hline $\mathrm{CO}$ & & 1,853 & $x$ & 106,667 & \\
\hline C5 & & 1,787 & $x$ & 101,000 & $x$ \\
\hline C10 & & 1,787 & $x$ & 102,000 & $x$ \\
\hline \multirow[t]{10}{*}{$\mathrm{C} 20$} & & 1,740 & $x$ & 94,667 & $x$ \\
\hline & & \multicolumn{3}{|c|}{ Diferencia } & \\
\hline & \multirow{2}{*}{$\begin{array}{c}\text { Contraste } \\
\qquad \bar{x}\end{array}$} & & & $\mathrm{SO}_{2}$ & \\
\hline & & $t=0$ & 34458 & $t=8,73435$ & \\
\hline & C0 - C05 & & ${ }^{*} 0,067$ & 5,667 & \\
\hline & C0 - C10 & & ${ }^{\star} 0,067$ & 4,667 & \\
\hline & С0 - C20 & & ${ }^{*} 0,113$ & *12,000 & \\
\hline & C05 - C10 & & 0,000 & $-1,000$ & \\
\hline & C05 - C20 & & 0,047 & 6,333 & \\
\hline & C10 - C20 & & 0,047 & 7,333 & \\
\hline
\end{tabular}


En general, el nivel de emisiones de CO para las mezclas es inferior que para el combustible diésel en todos los casos (Fig. 8). Este resultado puede ser atribuido al aumento de la cantidad de oxígeno disponible para la combustión con la adición de OCR al diésel, lo cual favoreció el proceso de oxidación en la cámara de combustión y redujo las emisiones de CO en los gases de escape (Yadav et al., 2016; Chuah et al., 2015; Varuvel et al., 2012). Resultados similares a los reportados por Cheikh et al. (2016), quienes establecieron que, a altas velocidades y cargas máximas, se generó una disminución de 8,2\% en las emisiones de CO de mezclas de biodiésel de cocina usado/ diésel al $50 \%$ vol., en comparación con el combustible diésel de referencia, la cual atribuyen al aumento en la concentración de oxígeno en el combustible y la temperatura de combustión en la cámara. Así mismo, Abedin et al. (2016) reportan que el incremento en el contenido de oxígeno en mezclas de biodiésel de palma/ diésel y b de laurel de Alejandría ( $20 \%$ vol.) /diésel, a velocidades altas, elevan la temperatura y tasa de combustión disminuyendo la emisión de $\mathrm{CO}$ en los gases de escape en 19 y $43 \%$ respectivamente, en relación al combustible diésel.

A partir del análisis de varianza (Tabla 6) y el contraste de medias (Tabla 5), se determinó que la concentración de $\mathrm{CO}$ en los gases de combustión del diésel y las mezclas ternarias, presenta diferencias significativas.

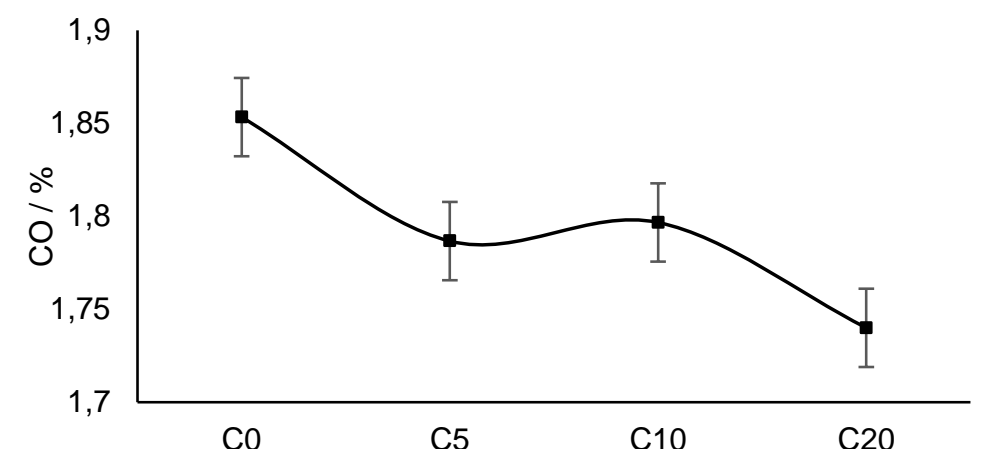

Fig. 8: Concentración de monoxido de carbono en los gases de escape.

Tabla 6: Análisis de varianza para la emisión de $\mathrm{CO}$ en función del tipo de combustible.

\begin{tabular}{llllll}
\hline Fuente & Suma de Cuadrados & Gl & Cuadrado Medio & Razón-F & Valor-P \\
\hline Entre grupos & 0,0195667 & 3 & 0,00652222 & 13,04 & 0,0019 \\
Intra grupos & 0,004 & 8 & 0,0005 & & \\
Total (Corr.) & 0,0235667 & 11 & & & \\
\hline
\end{tabular}

Por otro lado, aunque se esperaría un aumento en la concentración de $\mathrm{CO}_{2}$ en los gases de escape, al promover una mejora en el proceso de combustión con la adición de compuestos oxigenados al combustible (Özener et al., 2014; Shirneshan, 2013), las emisiones de $\mathrm{CO}_{2}$ en función del tipo de mezcla no presentaron una tendencia definida (Fig. 9). Estadísticamente el tipo de mezcla ternaria no influyó de forma significativa sobre la concentración de $\mathrm{CO}_{2}$ en los gases de escape (Tabla 7).

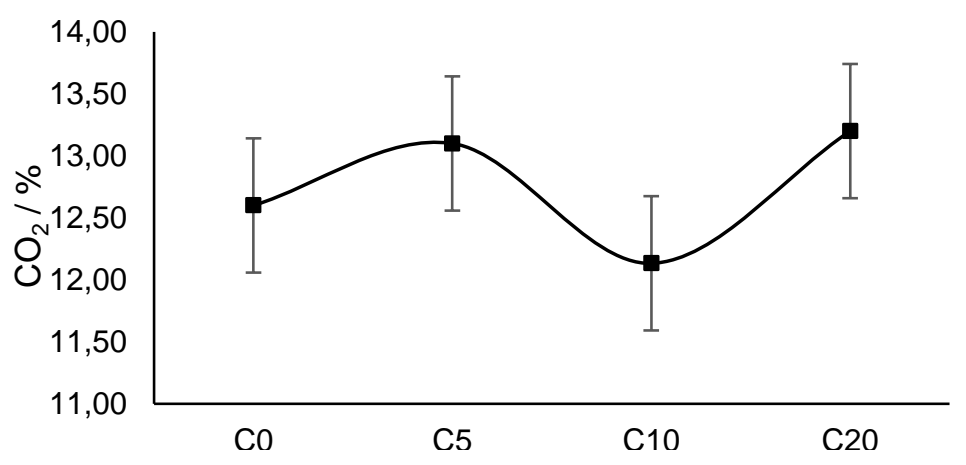

Fig. 9: Concentración de dioxido de carbono en los gases de escape.

Tabla 7: Análisis de varianza para la emisión de $\mathrm{CO}_{2}$ en función del tipo de combustible.

\begin{tabular}{llclcc}
\hline Fuente & Suma de Cuadrados & Gl & Cuadrado Medio & Razón-F & Valor-P \\
\hline Entre grupos & 2,1825 & 3 & 0,7275 & 2,20 & 0,1659 \\
Intra grupos & 2,64667 & 8 & 0,330833 & & \\
Total (Corr.) & 4,82917 & 11 & & & \\
\hline
\end{tabular}


A la luz de los resultados, la OCR podría ser considerada como una opción ambientalmente amigable para su uso como combustible en mezclas con diésel comercial. La reducción de gases contaminantes emitidos a la atmosfera y la mejora del comportamiento de flujo en frio del combustible, además de reducir la dependencia de combustibles fósiles, tendría un impacto positivo en el medio ambiente y posibilitaría la disminución de problemas como el taponamiento de filtros, conductos e inyectores a bajas temperaturas; sin embargo, el incremento en la viscosidad del combustible, limitaría su uso en aplicaciones automotrices pues puede afectar el desempeño del motor, al disminuir la potencia entregada por la máquina, debido a la dificultad para la inyección y atomización del combustible, sin considerar la liberación a futuro de los depósitos de carbón acumulados en el motor que podrían obstruir los filtros del combustible.

\section{CONCLUSIONES}

De acuerdo con las propiedades fisicoquímicas del diésel y sus mezclas con oleorresina de copaiba refinada (OCR), se estableció que la densidad y viscosidad del aceite incrementaron al aumentar la relación de mezcla OCR/diésel. La incorporación de OCR en las mezclas, mejoró los puntos de inflamación y el comportamiento de flujo en frio del combustible. El punto de fluidez en todos los casos se redujo a $-3^{\circ} \mathrm{C}$ y los puntos de inflamación se incrementaron hasta en $15^{\circ} \mathrm{C}$ respecto al combustible de referencia. Por otro lado, con la adición de $20 \%$ vol. de OCR al diésel, disminuyó la concentración de $\mathrm{SO}_{2}$ y $\mathrm{CO}$ en los gases de escape en 11 y $6 \%$ respectivamente. Se determinó que la adición de OCR al aceite combustible generó pérdidas de potencia en el motor de hasta el 19\%. La viscosidad ligeramente superior de las mezclas ternarias generó un incremento en la energía necesaria para bombear el aceite, ocasionando una disminución en la potencia entregada por el generador.

\section{AGRADECIMIENTOS}

Este estudio hace parte del proyecto de investigación INV-ING-2122, financiada por la Vicerrectoría de Investigaciones de la Universidad Militar Nueva Granada. Vigencia 2016.

\section{REFERENCIAS}

Abedin, M.J., M.A. Kalam y otros cinco autores, Production of biodiesel from a non-edible source and study of its combustion, and emission characteristics: A comparative study with B5, doi: 10.1016/j.renene.2015.11.027, Renew. Energy, 88, 20-29 (2016)

Ali, O.M., R. Mamat, N.R. Abdullah y A.A. Abdullah, Analysis of blended fuel properties and engine performance with palm biodiesel-diesel blended fuel, doi: 10.1016/j.renene.2015.07.103, Renew. Energy, 86, 59-67 (2016)

Ali, O.M., T. Yusaf y otros tres autores, Influence of Chemical Blends on Palm Oil Methyl Esters' Cold Flow Properties and Fuel Characteristics, doi: 10.3390/en7074364, Energies, 7, 4364-4380 (2014)

Altun, Ş. y M. Lapuerta, Properties and emission indicators of biodiesel fuels obtained from waste oils from the Turkish industry, doi: 10.1016/j.fuel.2014.03.024, Fuel, 128, 288-295 (2014)

ASTM D130-12, Standard, American Society for Testing and Materials, Standard Test Method for Corrosiveness to Copper from Petroleum Products by Copper Strip Test, West Conshohocken, PA (2012)

ASTM D2983-04, American Society for Testing and Materials, Standard Test Method for Low-Temperature Viscosity of Lubricants Measured by Brookfield Viscometer, West Conshohocken, PA (2004)

ASTM D92-05a, American Society for Testing and Materials, Standard test method for flash and fire points by Cleveland open cup tester, West Conshohocken, PA (2005)

ASTM D97-05c, American Society for Testing and Materials, Standard test method for pour point of petroleum products, West Conshohocken, PA (2005)

Atmanli, A., E. Ileri, B. Yuksel y N. Yilmaz, Extensive analyses of diesel-vegetable oil-n-butanol ternary blends in a diesel engine, doi: 10.1016/j.apenergy.2015.01.071, Appl. Energy, 145, 155-162 (2015)

Bardají, D.K., J.J. Da Silva y otros díez autores, Copaifera reticulata oleoresin: Chemical characterization and antibacterial properties against oral pathogens, doi: 10.1016/j.anaerobe.2016.04.017, Anaerobe, 40, 18-27 (2016)

Cheikh, K., A. Sary y otros tres autores, Experimental assessment of performance and emissions maps for biodiesel fueled compression ignition engine, doi: 10.1016/j.apenergy.2015.10.042, Appl. Energy, 161, 320-329 (2016)

Chen, F., H. Al-Ahmad y otros cinco autores, Within-plant distribution and emission of sesquiterpenes from Copaifera officinalis, doi: 10.1016/j.plaphy.2009.07.005, Plant Physiol. Biochem., 47, 1017-1023 (2009)

Chuah, L.F., A.R.A. Aziz y otros cuatro autores, Performance and emission of diesel engine fuelled by waste cooking oil methyl ester derived from palm olein using hydrodynamic cavitation, doi:1007/s10098-015-0957-2, Clean Technol. Environ. Policy, 17, 2229-2241(2015) 
Corsini, A., A. Marchegiani y otros tres autores, Vegetable Oils as Fuels in Diesel Engine, Engine Performance and Emissions, doi: 10.1016/j.egypro.2015.12.151, Energy Procedia, 81, 942-949 (2015)

Da Silva, W.G., N. Cortesi y P. Fusari, Copaiba oleoresin: evaluation of the presence of polycyclic aromatic hydrocarbons (PAHs), doi: 10.1590/S1984-82502010000300025, Braz. J. Pharm. Sci., 46, 597-602(2010)

Dhorm Pimentel de Moraes, A.R., G.D. Tavares y otros tres autores, Effects of nanoemulsions prepared with essential oils of copaiba- and andiroba against Leishmania infantum and Leishmania amazonensis infections, doi: 10.1016/j.exppara.2018.03.005, Exp. Parasitol., 187, 12-21(2018)

Espitia, A., A. Delgado y W. Aperador, Oleorresina de copaiba como materia prima para la producción de biodiésel, doi:10.20937/rica.2018.34.02.12, Rev. Int. Contam. Ambie, 34(2), 317-329 (2018)

Habibullah, M., H.H. Masjuki y otros cinco autores, Potential of biodiesel as a renewable energy source in Bangladesh, doi: 10.1016/j.rser.2015.04.149, Renew. Sustain. Energy Rev., 50, 819-834 (2015)

Lafont, J.J., M.S. Páez e Y.C. Torres, Análisis Químico de Mezclas Biodiesel de Aceite de Cocina Usado y Diesel por Espectroscopia Infrarroja, doi: 10.4067/S0718-07642011000400005, Inf. Tecnol., 22, 35-42 (2011)

Leevijit, T., G. Prateepchaikul y otros tres autores, Comparative properties and utilization of un-preheated degummed/esterified mixed crude palm oil-diesel blends in an agricultural engine, doi: 10.1016/j.renene.2016.08.047, Renew. Energy, 101, 82-89 (2017)

Morone, P. y L. Cottoni, Biofuels: Technology, economics, and policy issues; In Handbook of Biofuels Production, $2^{a}$ Ed., 61- 83 (R. Luque, C. Lin, K. Wilson, J. Clark, Eds.). Woodhead Publishing, Nueva Delhi, India (2016)

Özener, O., L. Yüksek, A.T. Ergenç y M. Özkan, Effects of soybean biodiesel on a DI diesel engine performance, emission and combustion characteristics, doi: 10.1016/j.fuel.2012.10.081, Fuel, 115, 875-883 (2014)

Qi, D.H., K. Yang, D. Zhang y B. Chen, Combustion and emission characteristics of diesel-tung oil-ethanol blended fuels used in a CRDI diesel engine with different injection strategies, doi: 10.1016/j.applthermaleng.2016.09.157, Appl. Therm. Eng., 111, 927-935 (2017)

Rakopoulos, D.C., C.D. Rakopoulos y otros tres autores, Influence of properties of various common bio-fuels on the combustion and emission characteristics of high-speed DI (direct injection) diesel engine: Vegetable oil, bio-diesel, ethanol, n-butanol, diethyl ether, doi: 10.1016/j.energy.2014.06.032, Energy, 73, 354-366 (2014)

Reddy, M.S., N. Sharma y A.K. Agarwal, Effect of straight vegetable oil blends and biodiesel blends on wear of mechanical fuel injection equipment of a constant speed diesel engine, doi: 10.1016/j.renene.2016.07.072, Renew. Energy, 99, 10081018 (2016)

Shirneshan, A., $\mathrm{HC}, \mathrm{CO}, \mathrm{CO}_{2}$ and $\mathrm{NO}_{x}$ Emission Evaluation of a Diesel Engine Fueled with Waste Frying Oil Methyl Ester, doi: 10.1016/j.sbspro.2013.04.033, Procedia - Soc. Behav. Sci., 75, 292-297 (2013)

Sitepu, I.R., L.A. Garay y otros cinco autores, Oleaginous yeasts for biodiesel: Current and future trends in biology and production, doi: 10.1016/j.biotechadv.2014.08.003, Biotechnol. Adv., 32, 1336-1360 (2014)

Suh, H.K. y C.S. Lee, A review on atomization and exhaust emissions of a biodiesel-fueled compression ignition engine, doi: 10.1016/j.rser.2015.12.329, Renew. Sustain. Energy Rev., 58, 1601-1620 (2016)

Tobouti, P.L., T.C. de Andrade Martins, T.J. Pereira y M.C. Mussi, Antimicrobial activity of copaiba oil: A review and a call for further research, doi: 10.1016/j.biopha.2017.07.092, Biomed. Pharmacother., 94, 93-99 (2017)

Varuvel, E.G., N. Mrad, M. Tazerout y F. Aloui, Experimental analysis of biofuel as an alternative fuel for diesel engines, doi: 10.1016/j.apenergy.2012.01.067, Appl. Energy, 94, 224-231 (2012)

Veiga junior, V.F. y A.C. Pinto, The Copaifera L. genus, doi: 10.1590/S0100-40422002000200016, Quím. Nova, 25, 273$286(2002)$

Yadav, A.K., M.E. Khan, A.M. Dubey y A. Pal, Performance and emission characteristics of a transportation diesel engine operated with non-edible vegetable oils biodiesel, doi: 10.1016/j.csite.2016.08.001, Case Stud. Therm. Eng., 8, 236-244 (2016) 